\title{
Colour Doppler valvar and subvalvar flow diameter imaging versus echo score in mitral stenosis: comparison with type of surgery
}

Colette Veyrat, Denis Pellerin, Dominique Sainte Beuve, Fabrice Larrazet, Daniel Kalmanson, Serge Witchitz

\begin{abstract}
Objective-To compare the value of echo score with that of Doppler subvalvar flow broadening in deciding the type of mitral stenosis surgery.

Patients-30 patients, mean age 47 years, with severe stenosis undergoing surgery were divided into two groups according to type of surgery: open heart commissurotomy (group $1, n=12$ ), or prosthesis (group 2, $\mathbf{n}=18$ ). A control group of 10 patients with prosthesis served as reference, representing mild stenosis without subvalvar connection.
\end{abstract}

Methods-For echo, the score proposed by Wilkins for cross sectional imaging was used. For Doppler, the flow diameters were measured in $\mathrm{cm}$ by an independent examiner from the long axis view in early diastole at two levels: (1) at the level of the stenosis (origin flow diameter), and (2) $1.5 \mathrm{~cm}$ downstream from the stenosis in the left ventricle (subvalvar flow diameter) with calculation of a Doppler ratio relating these two measurements, expressed as a percentage of broadening. Diagnostic value was compared for both procedures.

Results-There was no significant difference in age, mitral valve areas, or haemodynamics for the two groups. Mean values (SD) were: echo score: group 1, 9.83 $(1 \cdot 26) v$ group 2, 10.8 (8.1), NS; Doppler ratio \%: group 1,44 (24) $v$ group 2,12 (21) (P < 0.001); control group: 69 (15). The per cent diagnostic value for an open heart commissurotomy of respective cut off points was: Doppler ratio $>25 \%$ (range 71\% to 87\%); echo score < 10 (range $50 \%$ to $75 \%$ ).

Conclusions-The new Doppler ratio diagnostic value agreed better with surgical management, repair or prosthesis, in this study. Thus, it appears to better reflect the subvalvar involvement and changes in kinetics than the echo score alone. This easy Doppler method might become a routine examination for follow up of patients with open heart commissurotomy, to avoid performing repeated transoesophageal echocardiography.

(Heart 1996;75:486-491)

Keywords: mitral stenosis; Doppler imaging; subvalvar flow diameters; surgery
In mitral stenosis the choice between open heart commissurotomy and prosthetic insertion is not always reliably assessed from the echo score according to the Wilkins criteria. ${ }^{1}$ Mitral leaflet involvement is usually well defined, but there are still limitations in defining subvalvar structure involvement, even with the help of transoesophageal echography. ${ }^{2}$ Preoperative diagnosis ${ }^{3}$ and the later follow up of patients with open heart commissurotomy would also benefit from a completely non-invasive and repeatable procedure, rather than from routinely repeated transoesophageal examinations. Since in most cases the status of the subvalvar apparatus is the key to deciding the type of surgery, we hypothesised that flow information drawn from colour Doppler jet imaging, more specifically the subvalvar broadening of the jet downstream of the stenosis, might be an indirect marker of the degree of subvalvar involvement. We therefore compared the echo score and the Doppler values according to the final surgical decision.

\section{Methods}

We prospectively studied 34 patients with mitral stenosis, diagnosed as severe from catheterisation data before surgery. Of these, four were excluded for lack of required echoDoppler recording plane. There were 30 patients (18 females, 12 males, mean age 47 (SD 13) years), with a mean mitral valve area of $1.04(0 \cdot 19) \mathrm{cm}^{2}$ at catheterisation. Five patients had atrial fibrillation; the rest were in sinus rhythm. All patients were in functional class II or III, except two in class IV. Ten patients had mild mitral regurgitation as shown by angiography. Patients were examined from two days to three weeks before surgery.

We also studied 10 patients with a normally functioning mitral bioprosthesis (three of Ionescu-Shilley type and seven of CarpentierEdwards type), providing a control group of patients without any subvalvar connection.

\section{ECHO-DOPPLER EXAMINATION}

The equipment consisted of a wide angle, phased array, cross sectional scanner and a colour Doppler flow imaging display with a 2.0 or $2.5 \mathrm{MHz}$ dual frequency tranducer (Acuson $128 \mathrm{XP}$ ). We always used a regional enhancement selection (RES), where the maximum frame rate is optimised for depth with an internally programmed number of bursts 


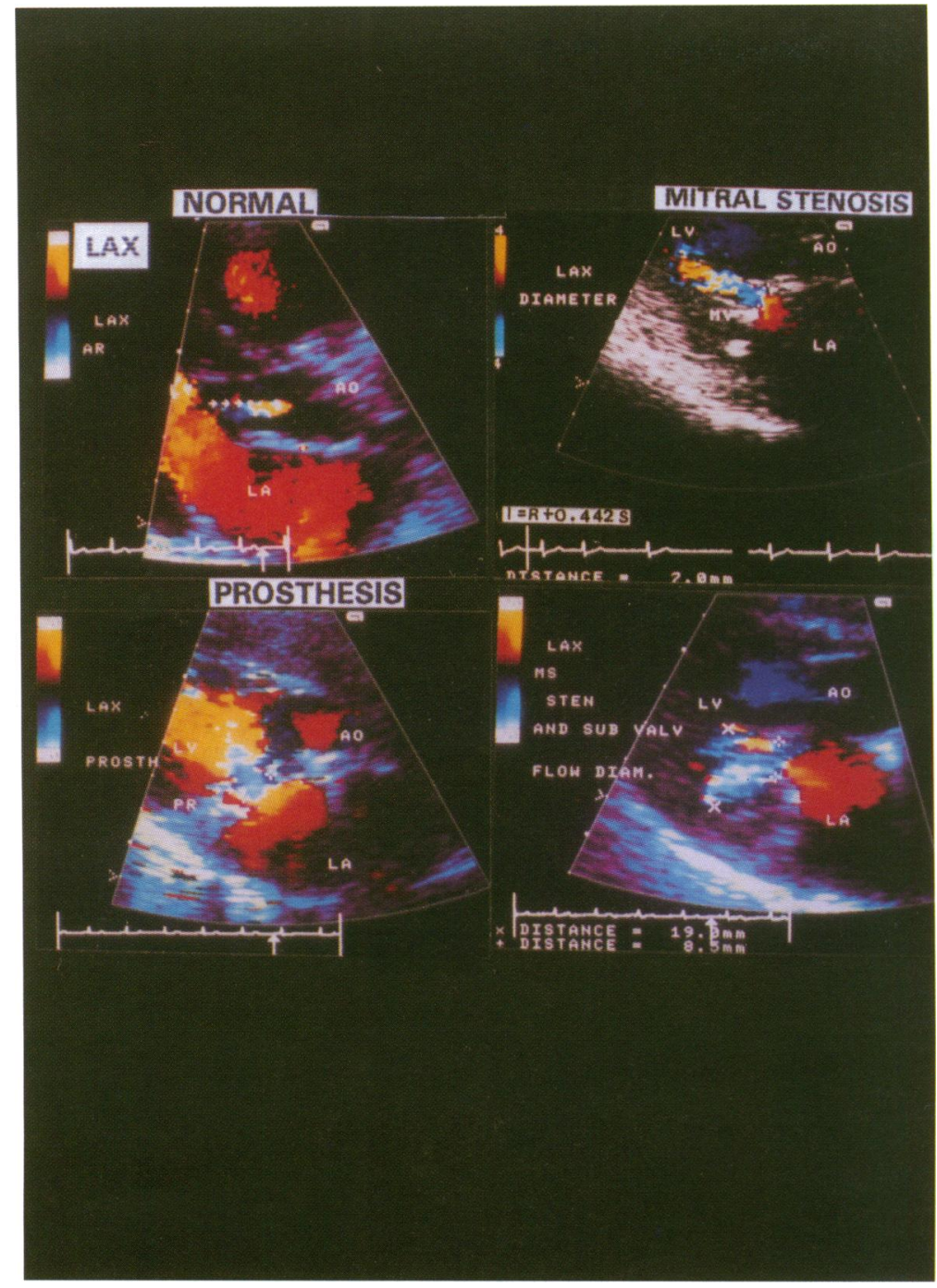

Figure 1 Comparative study of the flow diameters in the long axis view (valvar and subvalvar levels) in a patient with a normal mitral valve, in a control patient with bioprosthesis, and in two patients with mitral stenosis. Top left: this patient with mild aortic regurgitation has been chosen on purpose, to show that the aortic regurgitant jet (small white arrows) did not create difficulty in analysing the mitral flow in early diastole; flow is slightly accelerated without changes in diameter. Bottom left: with a bioprosthesis there is aliasing at the level of the prosthesis, then flow expands widely beyond the prosthesis. Top right: mitral stenosis with severe subvalvar lesions: no broadening of the flow diameter is seen beyond the stenosis, which is checked by the occurrence of aliasing (cross sectional image gated at the peak pressure gradient, $R$ wave of the electrocardiogram (cross sectional image gated at the peak pressure gradient, $R$ wave of the electrocardiogram
$+0.442 \mathrm{~s}^{5}$ ). This patient underwent prosthetic replacement. Bottom right: the Doppler ratio is 60\%; this patient had moderate subvalvar lesions, as confirmed by later open heart commissurotomy. $A O$, aortic orifice; $A R$, aortic regurgitation; diam, diameter; $L A, L V$, left atrium and ventricle; $L A X$, long axis view; $M S$, mitral stenosis; $M V$, mitral valve; $P R$, prosth, bioprosthesis; sub valv, subvalvar; sten, stenosis.

per scan line. The level of gain was fixed just below that entailing noise for standard echo and for colour (range 50-60) with medium filtering. The cross sectional and $M$ mode standard echo and colour images were stored on videorecorders (3/4 Umatic or $\frac{1}{2}$ inch VHS Pal Secam videotapes) and photographed with an incorporated camera $(24 \times 36 \mathrm{~mm}$ slides $)$.

The procedure consisted of two sequential parts, echocardiography and Doppler, both performed in the long axis view by separate observers, blind to each other. Patients were examined in the left lateral recumbent position.

\section{Colour Doppler imaging}

The site of the stenosis was identified by checking the start of aliasing, occurring after a red accelerated flow on the atrial side of the annulus on the cross sectional image, ${ }^{4}$ in a long axis plane. We visualised the stenotic jet from its origin at the mitral orifice downstream at the subvalvar structure level in the left ventricle (fig 1). The subvalvar flow changes could also be documented on colour $M$ mode recordings (fig 2).

The following variables were measured in early diastole ${ }^{5}$ (fig 3): (1) the smallest aliased jet flow diameter in $\mathrm{mm}$ at the site of the stenosis (orifice flow diameter); (2) in the left ventricle, the subvalvar flow diameter in $\mathrm{mm}$, $1.5 \mathrm{~cm}$ downstream of the orifice. The following ratio represented the percentage of flow broadening in the left ventricle:

(Subvalvar flow diameter - orifice flow diameter)/orifice flow diameter $\times 100$

The same procedure was applied to the control group of patients with prosthesis.

\section{Echocardiographic examination}

By a slight tilting of the transducer, as in the Doppler examination, the long axis planes showed the centre and the commissural areas of the mitral apparatus (fig $1 \mathrm{in}^{3}$ ). The changes in valve mobility, thickness, calcified deposits, and subvalvar structures were scored (table 2 in $^{1}$ ) with a score ranging from 0 for a normal appearance to 16 for maximum involvement. These changes were observed from long axis cross sectional views (fig 4, top), and could be further analysed on $M$ mode recordings (fig 4 , bottom). Since the study was limited to a comparison of the value of echo changes in mitral structures versus flow information, other echoDoppler measurements that were studied were not taken into account in the results reported here.

\section{STATISTICAL STUDY}

The surgical management was independent of the results of the variables studied (echo score or Doppler ratio). The interpretative variability was studied blindly from two readings performed by one observer at a three week interval (intraobserver) and by two observers (interobserver), both for colour flow diameters and for standard echo mitral structures. The differences within groups were expressed as per cent difference: reading 1 minus reading $2 /$ mean of both readings $\times 100$. All values are expressed as means (SD). When differences between values were found (by analysis of variance), their significance was assessed either by an unpaired Student $t$ test or by nonparametric tests $(P<0.05$ level). The diagnostic value of echo-Doppler data was assessed as recommended by Yerushalmy. ${ }^{6}$

\section{Results}

Patients with mitral stenosis were divided into two groups according to type of surgery: open heart commissurotomy in 12 patients (group 1) and prosthetic insertion in 18 patients (group 2), (table 1).

\section{GENERAL FINDINGS}

There was no significant difference between the groups in age [group 1, 47 (14) years; 


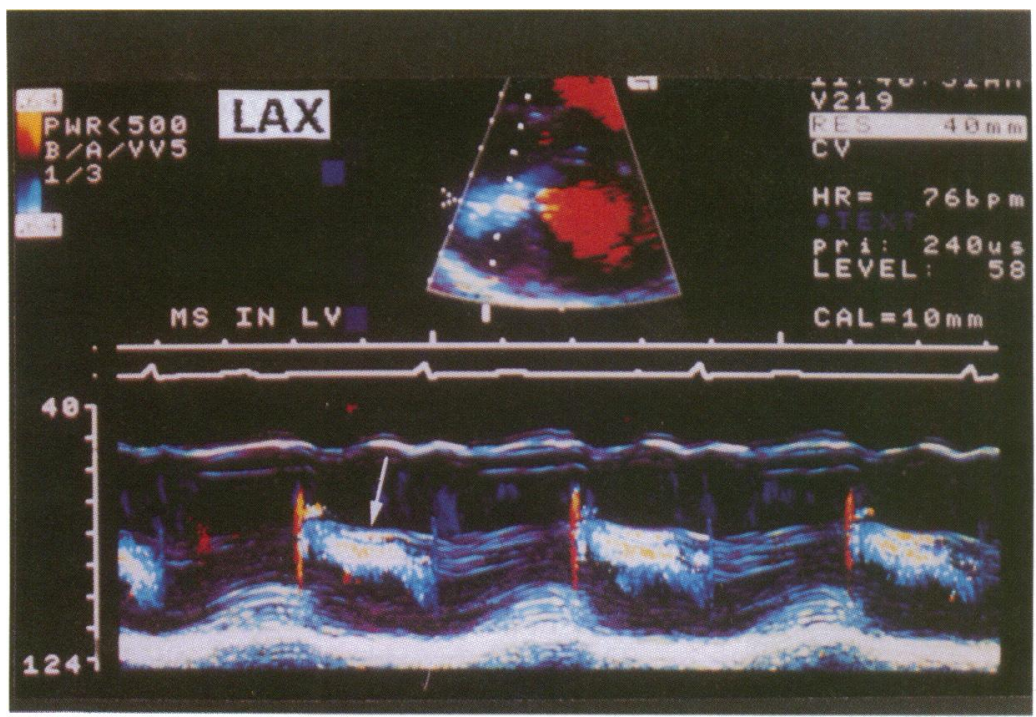

Figure 2 Colour $M$ mode recording in mitral stenosis with severe subvalvar lesions: the lack of broadening (oblique white arrow) may also be shown on this type of recording, locating the $M$ line further than the mitral orifice. In this example there is also loss of autonomous motion of the subvalvar structures which may be seen surrounding the jet and which follow the general heart motion during the entire cardiac cycle (compare with fig 4, bottom). Same abbreviations as in fig 1.

group 2, 47 (12) years], mitral valve area [group 1, 1.10 $(0.20) \mathrm{cm}^{2}$; group 2, 0.99 $\left.(0 \cdot 18) \mathrm{cm}^{2}\right]$, or general haemodynamics.

\section{ECHO-DOPPLER FINDINGS}

The feasibility was $100 \%$ for both procedures.

Interpretative variability

The per cent differences in intraobserver and interobserver measurements ranged from $2 \cdot 30$ $(2.69)$ to $2.49(3.16)$ for flow diameter, and from $3.75(5.15)$ to $4.73(5.03)$ for echo score, respectively. The interobserver difference between values found for flow diameters and echo score was significant $(P<0.03)$.

Table 1 Gender, age, mitral valve area (Gorlin), and Echo-Doppler data for patients with mitral stenosis, and type of surgery

\begin{tabular}{|c|c|c|c|c|c|c|}
\hline Patient No. & Sex & Age (years) & $M V A \mathrm{~cm}^{2}$ & Echo & $\begin{array}{l}\text { Doppler } \\
\text { ratio \% }\end{array}$ & Surgery \\
\hline 1 & $\mathbf{M}$ & 55 & $1 \cdot 1$ & 8 & 25 & $\mathrm{OHC}$ \\
\hline 2 & $\mathrm{~F}$ & 28 & 0.9 & 10 & -41 & PR \\
\hline 3 & $\mathrm{~F}$ & 19 & 0.9 & 12 & 0 & PR \\
\hline 4 & $\mathrm{~F}$ & 57 & $1 \cdot 27$ & 10 & 38 & $\mathrm{OHC}$ \\
\hline 5 & $M$ & 55 & 1.0 & 10 & 0 & $1 / \mathrm{OHC} 2 / \mathrm{PR}$ \\
\hline 6 & M & 70 & 0.9 & 12 & -10 & PR \\
\hline 7 & $\mathbf{M}$ & 58 & $1 \cdot 2$ & 11 & 40 & $\mathrm{OHC}$ \\
\hline 8 & $\mathrm{~F}$ & 47 & 1.0 & 8 & 58 & $\mathrm{OHC}$ \\
\hline 9 & $\mathrm{~F}$ & 37 & $1 \cdot 2$ & 9 & 25 & PR \\
\hline 10 & M & 49 & 0.9 & 14 & 0 & PR \\
\hline 11 & M & 18 & 0.8 & 10 & 50 & $\mathrm{OHC}$ \\
\hline 12 & $\mathrm{~F}$ & 68 & 1.3 & 9 & 0 & PR \\
\hline 13 & M & 39 & $1 \cdot 2$ & 8 & 75 & $\mathrm{OHC}$ \\
\hline 14 & $\mathrm{~F}$ & 59 & $1 \cdot 1$ & 9 & 50 & $\mathrm{PR}(\mathrm{Ca})$ \\
\hline 15 & $\mathrm{~F}$ & 42 & 0.9 & 8 & 22 & PR \\
\hline 16 & $\mathrm{~F}$ & 61 & $1 \cdot 0$ & 9 & 55 & $\mathrm{PR}(\mathrm{Ca})$ \\
\hline 17 & M & 62 & $1 \cdot 1$ & 12 & 33 & $\mathrm{OHC}$ \\
\hline 18 & M & 53 & $1 \cdot 3$ & 10 & 54 & $\mathrm{OHC}$ \\
\hline 19 & $\mathrm{~F}$ & 38 & 1.0 & 14 & 22 & PR \\
\hline 20 & $\mathrm{~F}$ & 55 & 1.2 & 8 & 0 & PR \\
\hline 21 & $\mathbf{F}$ & 42 & $1 \cdot 1$ & 11 & 16 & PR \\
\hline 22 & $\mathrm{~F}$ & 34 & 0.9 & 9 & 28 & PR \\
\hline 23 & M & 65 & 0.8 & 14 & 16 & PR \\
\hline 24 & $\mathrm{~F}$ & 40 & 1.2 & 10 & 0 & PR \\
\hline 25 & $\mathrm{~F}$ & 52 & $1 \cdot 2$ & 11 & 17 & PR \\
\hline 26 & $\mathrm{~F}$ & 38 & 0.6 & 12 & 0 & PR \\
\hline 27 & $\mathbf{F}$ & 35 & $1 \cdot 0$ & 10 & 89 & $\mathrm{OHC}$ \\
\hline 28 & M & 49 & 1.5 & 10 & 46 & $\mathrm{OHC}$ \\
\hline 29 & M & 51 & 0.9 & 14 & 16 & PR \\
\hline 30 & $\mathrm{~F}$ & 42 & 0.8 & 11 & 20 & $\mathrm{OHC}$ \\
\hline Mean & & 47 & $1 \cdot 04$ & 10 & 24 & \\
\hline SD & & 13 & $0 \cdot 19$ & $1 \cdot 8$ & 27 & \\
\hline
\end{tabular}

Ca, calcifications; F, female; M, male; MVA, mitral valve area; OHC, open heart commissurotomy; PR, prosthesis.

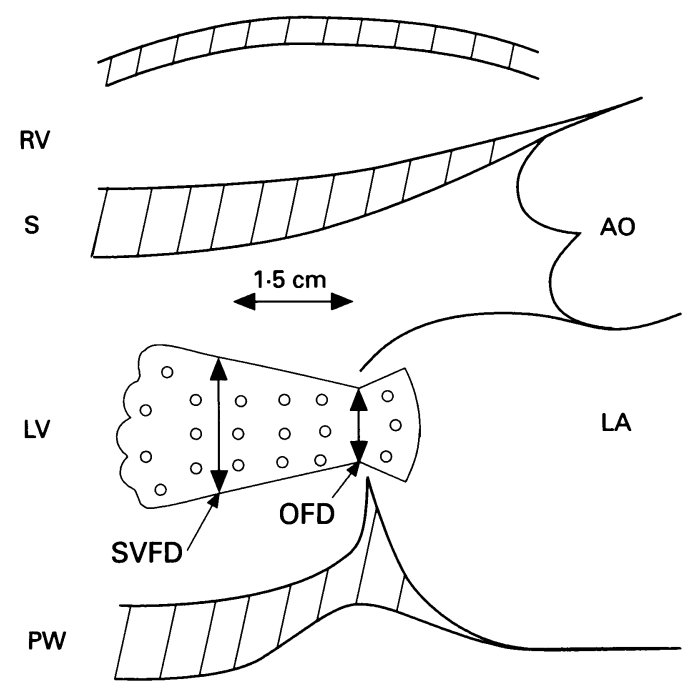

SVFD/OFD Doppler ratio (MS)

Figure 3 Mitral stenosis. Schematic representation of the Doppler flow diameter measurement (long axis view): the schema shows the trajectory of the jet (dotted area); the first measurement of the diameter is performed at the level of the stenosis where aliasing occurs; the subvalvar diameter is measured $1.5 \mathrm{~cm}$ downstream of this level and a ratio is calculated between both measurements (explanations in text). OFD, orifice flow diameter; $P W$, posterior wall; $R V$, right ventricle; $S$, septum; $S V F D$, subvalvar flow diameter.

\section{Colour Doppler findings}

The subvalvar part of the jet was observed in all cases but required some tilting of the transducer, with an approach intermediate between the long axis and the two chamber view in five cases. Figure 1 shows the findings for a subject with a normal valve, a patient with a mitral prosthesis, and for both patients with mitral stenosis. Figure 2 shows a colour $\mathrm{M}$ mode recording at the subvalvar level beyond the stenosis.

One prosthesis had to be inserted shortly after failure of repair (patient No 5).

Tables 1 and 2 list respectively the individual and mean values for the echo score and the flow diameters. The latter are illustrated in fig 5.

\section{Echocardiographic data}

Two patients (Nos 14 and 16) had completely calcified cusps, making the choice of a prosthetic insertion mandatory; however, there was a marked broadening of the subvalvar flow diameter, showing a possible lack of parallelism between the valvar and subvalvar involvement. We noted a discrepancy between the thickness of the subvalvar structures and their mobility in five cases during cross sectional examination. On $M$ mode recordings, these mobile subvalvar structures had an autonomous motion which differed from the general motion of the whole apparatus and could disappear from the $M$ mode image at certain periods of the cardiac cycle (fig 4).

The mean value and standard deviation of the echo score for the valvar (thickness and mobility) and subvalvar structures were as follows: valvar structures: group 1, 5.25 (0.75) $v$ group $2,6.00(1.23)(\mathrm{P}<0.05)$; subvalvar structures: group 1, 2.75 (0.82) v group 2, $2 \cdot 61(1 \cdot 03)(\mathrm{NS})$. 


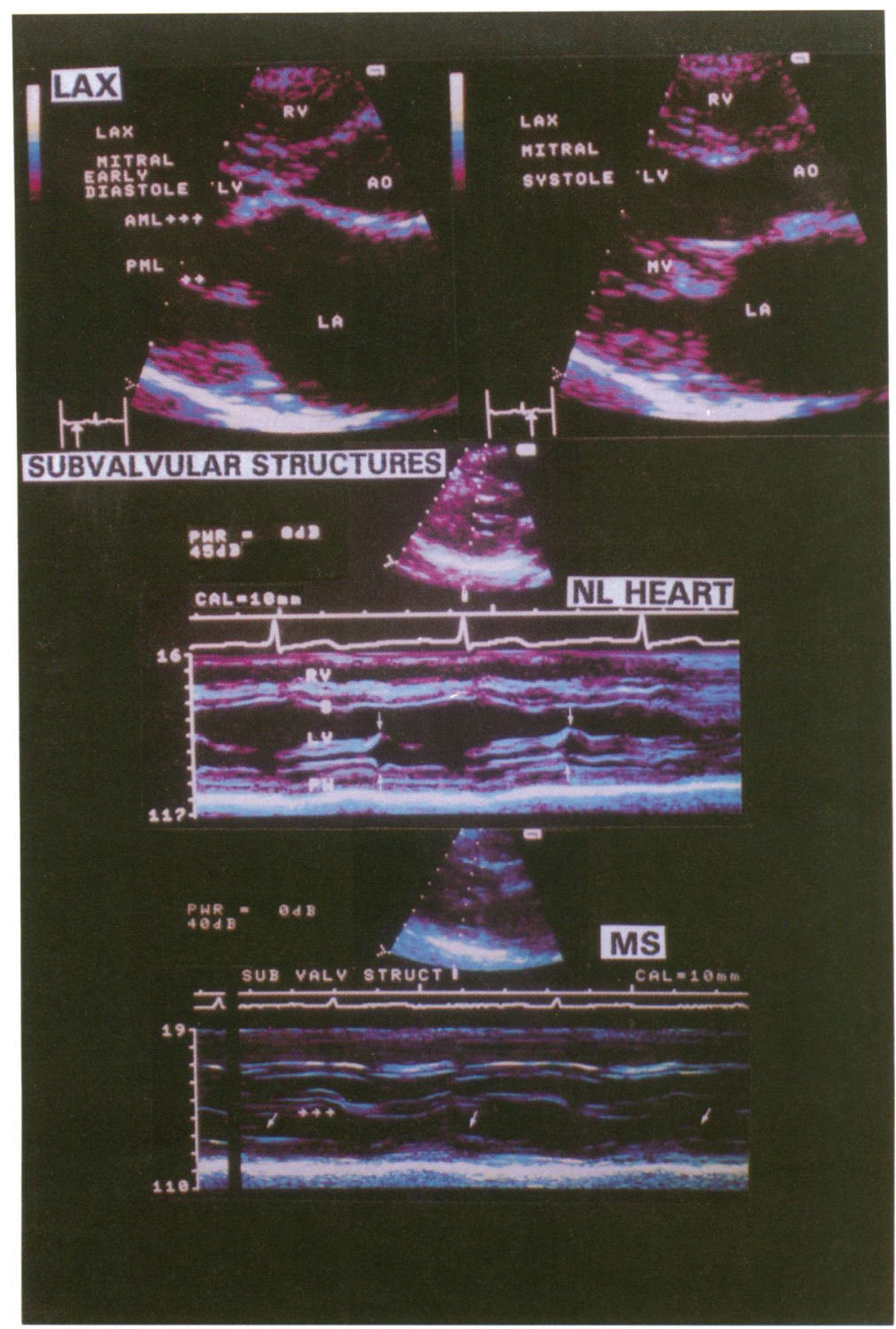

Figure 4 Mitral stenosis. Echocardiographic study, long axis view. Top: cross sectional image of moderately diseased mitral leaflets (small white arrows) with a doming anterior leaflet in early diastole; in systole, the changes in subvalvar structures are displayed near to the valves, at a distance from the septum. M mode recordings: these show that thickness depends on settings (increased transmitted power) and may be increased in normal individuals (middle traces, NL HEART), requiring a simultaneous analysis of the kinetics to rule out mitral stenosis (opposite diastolic motion, vertical white arrows). Bottom traces (MS): chordae pattern typical of mitral stenosis (horizontal white arrows), but oblique white arrows point to persistent autonomous posterior diastolic motion (compared with fig 2). AML, PLM, anterior and posterior mitral leaflets; NL, normal; sub valv struct, subvalvar structures; other abbreviations as in previous figures.

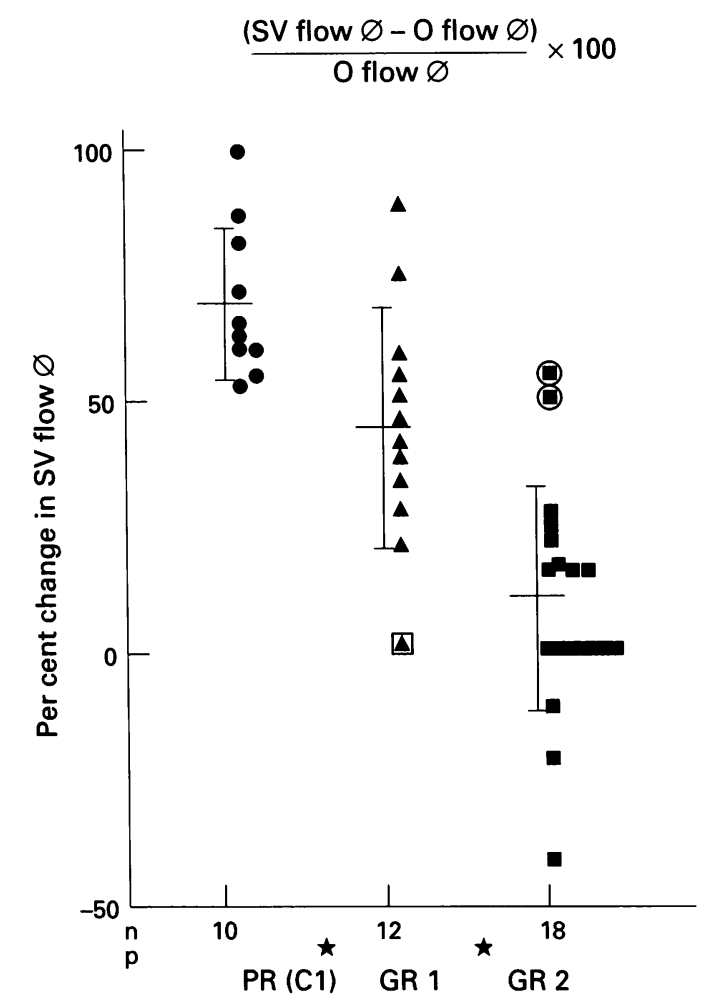

Figure 5 Diagram illustrating the results for the Doppler ratio. On the vertical axis, the per cent changes in the subvalvar flow diameter are shown for the control patients with mitral prosthesis (PR) and for patients with stenosis (group 1 with open heart commissurotomy; group 2 with prosthetic insertion). The triangle in a square in group 1 represents the patient who had a prosthesis following the repair; squares surrounded by circles in group 2 represent the patients with calcified leaflets requiring a prosthetic insertion in spite of moderate subvalvar lesions. The stars indicate significance between groups. C, controls; $G R$, group; $\varnothing$, diameter; $S V$, subvalvar; other abbreviations as in previous figures.

Table 3 Diagnostic value (\%) of an echo score $<10$ and of a Doppler ratio $>25 \%$ for prediction of an open heart commissurotomy

\begin{tabular}{lll}
\hline & Echo score & $\begin{array}{l}\text { Colour Doppler subvalvar } \\
\text { flow diameter ratio }\end{array}$ \\
\hline Sensitivity & 75 & 83 \\
Specificity & 50 & 77 \\
+ Predictive value & 50 & 71 \\
- Predictive value & 75 & 87 \\
Accuracy & 60 & 80 \\
\hline
\end{tabular}

DIAGNOSTIC VALUE

Table 3 gives the predictive value of echo versus the Doppler data for a prosthetic insertion versus open heart commissurotomy. Cut off points were 9 for the echo score and $25 \%$ for the subvalvar flow diameter broadening. The predictive value ranges from $50 \%$ to $75 \%$ for the former, and from $71 \%$ to $87 \%$ for the latter.

\section{Discussion}

COLOUR DOPPLER FLOW DIAMETER IMAGING The rationale of our hypothesis was that flow broadening downstream of the mitral orifice, might be a better-though indirect-dynamic marker of the subvalvar involvement than the 
changes in thickness seen on echocardiography. This hypothesis nevertheless does not take into account the effect of the orifice geometry on the downstream spread. The effect of an abrupt narrowing compared with a funnel shaped orifice on the vena contracta have been experimentally studied using the discharge coefficient, ${ }^{7}$ as has the influence of aortic valve morphology on the width of a regurgitant jet spreading into a large receiving chamber model. ${ }^{8}$ When the downstream chamber is bounded by a diseased subvalvar apparatus, it presumably generates resistive and friction forces, modifying left ventricular flow behaviour beyond the stenosis. Such conditions remain to be investigated in models. From a practical viewpoint, however, our study may offer an approximation of the pathophysiological consequences of a diseased subvalvar apparatus on flow: flow measurements were performed in early diastole, where the pressure gradient is highest and can be expected to move the subvalvar structures maximally.

Even if subvalvar changes extend to the tip of the papillary muscles, according to grade 3 of the Wilkins criteria, this does not preclude the persistence of some mobility. This subvalvar mobility is one of the main determinants of the chamber boundaries, where the jet may broaden. Conversely, lack of mobility should entail a more or less narrow funnel shaped stricture downstream. This additional stenosis should limit jet expansion in the subvalvar area of the left ventricle throughout diastole, as shown on colour $\mathrm{M}$ mode traces. Our findings that flow predicted the choice of mitral stenosis surgery better than echo information, and that there is a reasonable broadening of the stenotic jet in patients treated by open heart commissurotomy, are most likely to be due to the persistence of some subvalvar mobility in group 1, and are in favour of our hypothesis.

Postoperative changes in the Doppler flow diameter ratio should indicate a satisfactory subvalvar repair. Benefit from this easily repeatable examination during the postoperative follow up of open heart commissurotomy will require further study.

Some methodological issues have to be considered in relation to colour Doppler imaging. The use of the long axis rather than the apical approach, which has an optimal Doppler angle, may be questioned. The advantages were twofold: (1) it made the site of examination nearer to the chest, and the measurements were easy, quickly performed, and could be confirmed by colour $M$ mode recordings; (2) there was less confusion than with the apex view between the frequently associated aortic regurgitant jet and the mitral inflow jet. Another methodologically important point is to perform the flow diameter measurements in early diastole, ${ }^{5}$ to avoid flow mixing and to standardise interobserver measurements.

ECHO SCORE

Our study has a further area of interest: it prompts us to reconsider the limitations of the echo score. Only moderate reliability of the echo score has been shown, although it was found to be the best predictor of restenosis after valvoplasty. ${ }^{9}$ On the other hand, follow up of valvoplasty by stepwise multiple regression analysis ${ }^{10}$ failed to demonstrate a major role of total mitral morphology score as a predictor of the results of balloon dilatation at a six month follow up. Success rate was more related to the duration of the disease and to the size of the balloon. The Wilkins score was used in both studies, and this may partly explain these conclusions. Indeed, the subvalvar status, which is so important to consider for reconstructive surgery, is underscored in the grading, since it accounts for only one fourth of the total score in the preoperative assessment. The items retained by Wilkins for the subvalvar evaluation are thickness and length of involvement from the leaflets to the papillary muscles, exclusive of kinetics. Subvalvar shortening (grade 4) is very operator dependent in intermediate cases, since chordae tendinae length has not been serially measured on patients or in experiments. ${ }^{11}$ On the other hand, figures do not always guarantee reliability for assessing echo structures status, given the gain variations ${ }^{12}$ : a previous echo-surgical study ${ }^{3}$ failed to show the value of thickness measurements because of overlap in values among patients with increasing subvalvar involvement at surgery. Interobserver dependency is also high in thickness assessment. Gain and transmitted power also intervene; subvalvar structures may appear thickened even in a normal patient, as shown in fig 4. In our opinion, visual evaluation-recommended by Wilkins to avoid tedious measurements-is sufficient where criteria dealing with kinetics, that is, the persistence of an autonomous motion, are combined with thickening. $M$ mode recordings, having enhanced temporal resolution, should always be combined with cross sectional images. Disappearances from the image during some parts of diastole may be caused by this autonomous motion or by translational heart or respiratory movements. If so, similarly timed changes should apply to the mitral leaflets, which should always be analysed at the same time. Further evaluation of the echo score, updated for subvalvar kinetics, will be required. On the other hand, in patients 14 and 16 the value of some findings was obvious, such as entirely calcified leaflets, whatever the flow diameter broadening might be. This advantage of the Wilkins score for valvar tissue evaluation justifies its combination with the Doppler ratio.

So far as the control population is concerned, normals obviously cannot serve as controls, since there is hardly any acceleration at the site of a normal mitral annulus, and no change in flow diameter along the flow trajectory (fig 1). This is why we chose to use patients with a previously inserted mitral bioprosthesis, as such prostheses have a near normal inflow profile. This group provided a comparison with mild mitral stenosis and lack of subvalvar connection. The percentage increase in Doppler ratio clearly separated these from patients with severe mitral stenosis. 


\section{LIMITATIONS}

Our purpose was limited to the echo score and to Doppler flow diameter measurement, as compared according to type of surgery. Thus we did not take into account the other echo-Doppler or haemodynamic measurements or the duration of disease; as a whole, however, there was no significant difference in these variables. Differences in cardiac output could not explain variations in jet broadening.

An examination using one plane may seem unrepresentative of the complexity of the three dimensional nature of structures and flow phenomena. Since the aim of the study was to compare values of variables, we decided to retain a single echo plane to make our method of measurement clear and straightforward. Apical views may be more or less fore shortened, and short axis views lack clear landmarks for consistent measurement of variables at the given level of $1.5 \mathrm{~cm}$ beyond the stenosis. For best representation of the lesions, we systematically investigated the commissural areas by slightly tilting the transducer so as not to miss any asymmetrically diseased area.

In our study, echo and Doppler examinations were carried out independently, for obvious methodological reasons. The major calcifications could not be ignored and might have biased the results. However, predictive value still remained higher for flow information, even when the two patients with calcifications were omitted from the analysis.

Major aortic regurgitation might impede colour Doppler subvalvar measurement, and was excluded in our study. Atrial fibrillation requires averaged measurements, performed after a sufficiently long R-R interval. Optimal gain setting is easily recognised by experienced users.

Finally, this study obviously needs further confirmation on a larger cohort of patients, including balloon valvoplasty. Above all, the value of the flow criteria will need to be assessed during the follow up of patients with open heart commissurotomy. The true practical impact of the Doppler ratio on surgical management will only come from longitudinal studies on patients.

\section{CONCLUSIONS}

(1) Jet flow diameter measurement provided a better marker of subvalvar involvement and had a higher diagnostic value for open heart commissurotomy than the echo score. (2) An updated version of the echo score might correlate better with the type of surgery needed: for example, grading the subvalvar mobility separately from thickness would re-equilibrate the echo score by enhancing the role of the subvalvar status. (3) By coupling the updated echo score and the colour Doppler data, routine studies can be performed that are comparable with the efficient, but far more expensive, transoesophageal mitral imaging. This has benefits for the patient in terms of cost and convenience.

1 Wilkins GT, Weyman AE, Abascal VM, Block PC, Palacios IF. Percutaneous balloon dilatation of the mitral valve: an analysis of echocardiographic variables related to outcome and the mechanism of dilatation. Br Heart $f$ to outcome and the

2 Marwick TH, Torelli J, Obarski T, Casale PN, Stewart WJ. Assessment of the mitral valve splitability score by transAssessment of the mitral valve splitability score by trans-
thoracic and transesophageal echocardiography. $A m \mathcal{F}$ thoracic and transesop

3 Veyrat C, Villemot JP, Manin JP, Cabrol C, Kalmanson D. Anatomic and functional evaluation of pure and associated mitral stenoses using the echo-Doppler scanner technique. Ultrasound Med Biol 1983:9:1-17.

4 Veyrat C, Sainte Beuve D, Gourtchiglouian C, Legeais S, Kalmanson D. Quantification of left-sided valvular stenoses by color-Doppler imaging of jets. Angiology 1990;41:352-63.

5 Veyrat C, El Yafi W, Gourtchiglouian C, Bas S, Sainte Beuve D, Kalmanson D. Respective timing of maximal color Doppler jet areas and of peak velocity of jets in leftsided valvular jets. $\mathcal{A}$ Am Soc Echocardiogr 1991;4:258-66.

6 Yerushalmy J. Statistical problems in assessing methods of medical diagnosis, with special reference to $\mathrm{X}$-ray techniques. Public Health Rep 1947;62:1432-49.

7 Flachskampf FA, Weyman AE, Guerrero JL, Thomas JD. Influence of orifice geometry and flow rate on effective valve area: an in vitro study. $\mathcal{F}$ Am Coll Cardiol 1990;15: $1173-80$.

8 Taylor AL, Eichhorn EJ, Brickner ME, Eberhard RC. Aortic valve morphology: an important in vitro determinant of proximal regurgitant jet width by Doppler flow mapping. $₹ \mathrm{Am}$ Coll Cardiol 1990;16:405-12.

9 Abascal VM, Wilkins GT, Choong CY, Thomas JD, Palacios IF, Block PC, et al. Echocardiographic evaluation of mitral valve structure and function in patients followed for at least 6 months after percutaneous balloo

10 Reid CL, Otto CM, Davis KB, Labovitz A, Kisslo KB, McKay CR. Influence of mitral valve morphology on mitral balloon commissurotomy: immediate and sixmitral balloon commissurotomy: immediate and sixmonth results from the NHLBI ballo

11 Salisbury PF, Cross CE, Rieben PA. Chordae tendinae tension. Am F Physiol 1963;205:385-92.

12 Martin RP, Rakowski H, Kleiman JH, Beaver W, London E, Popp RL. Reliability and reproducibility of two dimensional echocardiographic measurement of the stenotic mitral orifice area. Am f Cardiol 1979:43:560-8. 